\title{
Totale Institutionen - Psychiatrien im 19. Jahrhundert am Beispiel der k.k. Provinzial-Irren-Heilanstalt Hall in Tirol
}

\author{
Dominique Karner \\ Wirtschafts- und Sozialgeschichte \\ eingereicht bei: ao. Univ.-Prof. ${ }^{\text {in }}$ Dr. in Elisabeth Dietrich-Daum \\ eingereicht im Semester: WS 2014/15 \\ Rubrik: PS-Arbeit
}

\begin{abstract}
Total Institutions - Mental Asylums in the $19^{\text {th }}$ Century

The following seminar paper is about mental asylums as „total institutions.” It analyzes the four important aspects of Erving Goffman's definition of a total institution. Finally, the paper discusses whether the psychiatry Hall can be outlined as one.
\end{abstract}

\section{Einleitung}

„Es gibt nichts Schockierenderes als Idiotie in der Hütte eines irischen Landarbeiters [...]. Werden ein kräftiger Mann oder eine Frau von den Beschwerden befallen, bleibt [den Familienmitgliedern] nichts anderes übrig, als ein Loch in den Boden der Hütte zu graben, nicht so tief, daß ein Mensch aufrecht darin stehen könnte, mit einem Lattengerüst darüber, damit er nicht herausklettern kann. Das Loch ist ungefähr einen Meter fünfzig tief; dort hinein reichen sie dem bedauernswerten Wesen die Mahlzeit, und dort stirbt es im allgemeinen."

Dieses aus dem Jahre 1817 stammende Zitat eines Mitglieds aus dem britischen Unterhaus stellt eine Skizzierung der Verwahrung eines psychisch Kranken in Irland dar, der in keiner Anstalt untergebracht werden konnte, sondern von seinen Familienmitgliedern zu Hause verwahrt wurde. Man kann sich vorstellen, dass diese oder zumindest eine

1 William P. Letchworth, zit. n. Edward Shorter, Geschichte der Psychiatrie, Berlin 1999, S. 13 f. 
ähnliche Art der Verwahrung vor dem Aufkommen der psychiatrischen Anstalten im 19. Jahrhundert auch in anderen Gebieten Europas eine gängige Methode dargestellt haben könnte, wie aus Edward Shorters Ausführungen hervorgeht. Er nennt diverse Fälle von Misshandlungen und menschenunwürdiger Behandlung von psychisch gestörten Menschen. Unter anderem berichtet er über einen 16-jährigen Jungen, der aufgrund seines „Irrsinns" 1798 von seinem Vater im Schweinestall angekettet und wie ein Tier gehalten wurde. ${ }^{2}$ Diese Umstände verdeutlichen zugleich den immensen Wert, den die Herausbildung von Orten der Verwahrung wie der Irren-Heilanstalt im 19. Jahrhundert mit sich brachte. Die Menschen fürchteten sich vor dem Anderssein und ein abnormales, nicht normgerechtes Verhalten wurde mit "autoritäre[r] Intoleranz"3 bestraft. Häusliche Gewalt gegenüber "geisteskranken“ Familienmitgliedern war laut Shorter bis ins 19. Jahrhundert keine Seltenheit. ${ }^{4}$ Mit der Gründung der ersten Anstalten gab es schließlich Institutionen mit Pflege- und Wartpersonal, das sich jener Individuen mit einer kranken Psyche annahm. Dennoch bedurfte es weiterer Jahrzehnte, bis eine optimale und gewaltfreie Behandlung in diesen Institutionen gewährleistet werden konnte.

Die folgende Proseminararbeit skizziert zunächst die historische Entwicklung der Psychiatrie bis zum 19. Jahrhundert. Im Anschluss daran werden die zentralen Inhalte des

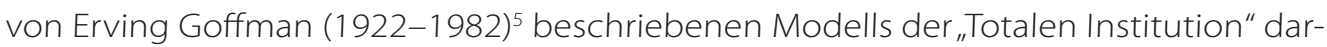
gestellt. Im nächsten Kapitel wird überprüft, ob jene vorgestellten Inhalte aus Goffmans „Asylums" auch auf das gewählte Beispiel der Provinzial-Irren-Heilanstalt zu Hall in Tirol zutreffen und in der Praxis Anwendung gefunden haben. Hierbei soll insbesondere auf den minutiös vorgegebenen Tagesablauf der Insassen und Insassinnen eingegangen werden. Abschließend soll eine Antwort auf die Frage, wie die InsassInnen selbst die in der Anstalt herrschenden strengen Reglementierungen wahrgenommen haben, formuliert werden. Der Untersuchungszeitraum umfasst dabei die Jahre 1830 bis 1900.

Goffmans Werk, das 1961 entstanden ist, zählt zu den Klassikern der Soziologie und ging aus seiner teilnehmenden Beobachtung am St. Elisabeths Hospital in Washington, D.C. von 1955/6 hervor. ${ }^{6}$ Es umfasst vier eigenständige Aufsätze, die einander ergänzen. Der erste Aufsatz mit dem Titel "On the Characteristics of Total Institution“ enthält eine allgemeine Untersuchung des Soziallebens in Orten der Verwahrung. Als veranschaulichende Beispiele setzt Goffman den Fokus auf psychiatrische Kliniken und Gefängnisse. Im zweiten Essay „The Moral Career of the Mental Patient" werden jene Faktoren untersucht, die auf die sozialen Beziehungen eines Individuums nach dessen

\footnotetext{
Shorter, Geschichte der Psychiatrie, S. 15.

Ebd., S. 14

Ebd., S. 15

Erving Goffman, Asylums. Essays on the Social Situation of Mental Patients and other Inmates, London 1991.

Falk Bretschneider/Martin Scheutz/Alfred Stefan Weiß, Machtvolle Bindungen - Bindungen voller Macht. Personal und Insassen in neuzeitlichen Orten der Verwahrung zwischen Konfrontation und Verflechtung, in: Falk Bretschneider/Martin Scheutz/Alfred Stefan Weiß (Hrsg.), Personal und Insassen von „Totalen Institutionen“ zwischen Konfrontation und Verflechtung (Geschlossene Häuser. Historische Studien zu Institutionen und Orten der Separierung, Verwahrung und Bestrafung 3), Leipzig 2011, S. 8.
} 
Einlieferung in eine totale Institution einwirken. In „The Underlife of a Public Institution“ werden die Insassen einer öffentlichen Institution selbst ins Licht gerückt. Der letzte Aufsatz "The Medical Model and Mental Hospitalization“ wiederum stellt das Personal einer psychiatrischen Anstalt in den Mittelpunkt.?

Als Quellengrundlage, die parallel zu Goffmans „Asylums" zur Herausarbeitung des Hauptteils dieser Arbeit genutzt wurde, diente die "Beschreibung"8 des ehemaligen Heilanstaltsdirektors Johann Tschallener (1783-1855) über die Irren-Heilanstalt Hall in Tirol. Tschalleners Ausführungen waren zielführend hinsichtlich der Beantwortung der Forschungsfrage, ob Goffmans Aspekte und Merkmale „Totaler Institutionen" in Bezug auf die Irren-Heilanstalt Hall in Tirol des 19. Jahrhunderts zutreffend sind. Ausgehend von dieser Frage wurde die These formuliert, dass die Haller Anstalt als „Totale Institution" angesehen werden kann.

Zuletzt sei noch auf das Werk "Psychiatrische Landschaften"9 sowie auf den Ausstellungskatalog „Ich lasse mich nicht länger für einen Narren halten“10 hingewiesen. Im erstgenannten Buch geht es um Psychiatriegeschichten, die die PatientInnen und das Personal psychiatrischer Einrichtungen in Tirol, Südtirol und im Trentino in den Mittelpunkt rücken. Die Historikerlnnen sprechen aber auch Einzelaspekte zur Geschichte der „'psychiatrischen Landschaft' im ,historischen Tirol' von 1830 bis zur Gegenwart"11an. Das Begleitbuch „Ich lasse mich nicht länger für einen Narren halten“ zur Ausstellung zur Geschichte der Psychiatrie in Tirol, Südtirol und im Trentino richtet den Fokus insbesondere auf die Patientinnen und Patienten der Anstalten, um die „Psychiatriegeschichte aus Patientenperspektive ${ }^{\prime 12}$ verfolgen zu können. Diese Lebensgeschichten waren sehr hilfreich, um die Frage zu beantworten, wie die Insassen der Irren-Heilanstalt Hall den streng reglementierten Anstaltsalltag empfunden haben.

\section{Die historische Entwicklung der Psychiatrie}

Bereits vor dem 18. Jahrhundert gab es in Europa Verwahranstalten (Gefängnisse, Asyle, Spitäler oder Armenhäuser), die nur für die Unterbringung von Geisteskranken bestimmt waren, aber keineswegs therapeutische Unterstützung boten. ${ }^{13}$ Das im 13. Jahrhundert in London gegründete Priory of St. Mary of Bethlem zählte beispielsweise

7 Goffman, Asylums, S. $11 \mathrm{f}$.

8 Johann Tschallener (Hrsg.), Beschreibung der k.k. Provincial-Irren-Heilanstalt zu Hall in Tirol mit Rücksicht auf die Statuten der Anstalt, auf die therapeutischen und psychologischen Grundsätze der Behandlung der Geisteskranken und ihre achtjährigen Resultate; mit 19 Krankengeschichten und verschiedenen Andeutungen zum Wohl dieser Unglücklichen; nebst einem Anhange über die Anlage von Zimmern für Irre und Tobende, Innsbruck 1842.

9 Elisabeth Dietrich-Daum/Hermann J.W. Kuprian/Siglinde Clementi/Maria Heidegger/Michaela Ralser (Hrsg.), Psychiatrische Landschaften. Die Psychiatrie und ihre Patientinnen und Patienten im historischen Raum Tirol seit 1830, Innsbruck 2011.

10 Maria Heidegger/Celia di Pauli/Lisa Noggler/Siglinde Clementi/Michaela Ralser/Elisabeth Dietrich-Daum/Hermann Kuprian (Hrsg.), Ich lasse mich nicht länger für einen Narren halten. Eine Ausstellung zur Geschichte der Psychiatrie in Tirol, Südtirol und im Trentino, Bozen 2012.

11 Dietrich-Daum et al. (Hrsg.), Psychiatrische Landschaften, S. 12.

12 Heidegger et al., Ich lasse mich, S. 10.

13 Shorter, Geschichte der Psychiatrie, S. 20 f. 
zu einem der ersten Krankenhäuser in Europa, das „psychisch Gestörte“ aufnahm und bis ins Jahr 1948 als städtische Irrenanstalt fungierte. ${ }^{14}$

Im Zuge der im 18. Jahrhundert aufkeimenden Idee, dass die Anstalt selbst eine therapeutische Funktion innehaben könnte, kam es zur Herausbildung der Psychiatrie. ${ }^{15}$ Den Behandlungsfokus legte man neben den medizinischen Anwendungen wie Aderlass, speziellen Diäten und der Verabreichung von Abführ- und pflanzlichen Arzneimitteln auf eine pädagogische Vorgehensweise, die Moral durch strikte Reglementierung, Arbeitszwang, Zucht und Ordnung vermitteln sollte, um geheilte PatientInnen in die Gesellschaft reintegrieren zu können. ${ }^{16}$ Die Psychiatrie nahm in der "Geschichte der Disziplin"17 angesichts der in den Anstalten herrschenden strikten Verordnungen eine entscheidende Rolle ein. Die Historikerin Andrea Chmielewski geht davon aus, dass die Ablehnung von "gefährlichen Irren“ durch die Hospitäler mitverantwortlich für die Gründung von Irrenanstalten im 18. Jahrhundert gewesen sei, da in den Spitälern keine spezielle Beaufsichtigung der "gefährlichen Irren" gewährt werden konnte. ${ }^{18}$ Michaela Ralser wiederum erachtet die Anstalten als sogenannte "Epiphänomene der bürgerlichen Revolutionen", ${ }^{19}$ die in Europa ihren Lauf nahmen und schreibt die Entstehung der Irrenanstalten im 18. und 19. Jahrhundert zugleich der "Medikalisierung der Irrenfrage ${ }^{\prime \prime 20} z u$.

Mit Beginn des 19. Jahrhunderts entstand schließlich die "moderne Krankenanstalt als klinisch-therapeutischer Versorgungsraum",21 die für das Heilen, das Verwahren und die Pflege psychisch Erkrankter sorgte. Im historischen Tirol übernahmen diese Aufgabe die beiden „'Landes-Irrenanstalten' in Hall und Pergine“22.

Einen Aufschwung erlebte die Irrenanstalt ab 1860, ${ }^{23}$ als sowohl die Anzahl an Einrichtungen selbst als auch die Zahl an InsassInnen drastisch zunahm. ${ }^{24}$ Stellt man den vielen anderen überfüllten Irrenanstalten ${ }^{25}$ jedoch die Haller Anstalt gegenüber, fällt auf, dass in Hall ein übermäßig hoher Patientenansturm durch strikte Maßstäbe positiv reguliert wurden, sodass es zu keiner Überfüllung kam. Diese bestanden unter ande-

14 Shorter, Geschichte der Psychiatrie, S. 18

15 Ebd., S. 23.

16 Elisabeth Dietrich-Daum/Maria Heidegger, Menschen in Institutionen der Psychiatrie, in: Dietrich-Daum et al. (Hrsg.), Psychiatrische Landschaften, S. 51.

17 Shorter, Geschichte der Psychiatrie, S. 61.

18 Alexandra Chmielewski, Staat und Irrenfürsorge. Badische Psychiatriereformen im 19. Jahrhundert, in: Zeitschrift für die Geschichte des Oberrheins 146 (1998), S. 437-453, hier S. 440 f.

19 Michaela Ralser, Das Subjekt der Normalität. Das Wissensarchiv der Psychiatrie: Kulturen der Krankheit um 1900, München 2010, S. 152.

20 Ebd.

21 Ebd., Im Vordergrund die Klinik. Das Beispiel der Innsbrucker Psychiatrisch-Neurologischen Universitätsklinik um 1900, in: Geschichte und Region/Storia e Regione 17 (2008), Heft 2, S. 135-145, hier S. 135.

22 Angela Griessenböck, Zur Geschichte der psychiatrischen Landschaft im Kronland Tirol: Die „Landes-Irrenanstalten" in Hall in Tirol und in Pergine, in: Eberhard Gabriel/Martina Gamper (Hrsg.), Psychiatrische Institutionen in Österreich um 1900, Wien 2009, S. 121-134, hier S. 122.

23 Heinz-Peter Schmiedebach, ZZerquälte Ergebnisse einer Dichterseele“. - Literarische Kritik, Psychiatrie und Öffentlichkeit um 1900, in: Heiner Fangerau/Karen Nolte (Hrsg.), Moderne Anstaltspsychiatrie im 19. und 20. Jahrundert. Legitimation und Kritik (Medizin, Gesellschaft und Geschichte 26), Stuttgart 2006, S. 259-281, hier S. 259.

24 Shorter, Geschichte der Psychiatrie, S. 60.

25 Überfüllung herrschte in vielen deutschen, französischen, aber auch englischen Anstalten: Shorter, Geschichte der Psychiatrie, S. 80-81. 
rem in der Differenzierung von heilbaren PatientInnen und „'Unheilbaren' [...] [sowie] ,blödsinnigen Individuen".26 Die zuletzt genannten Gruppen wurden von vornherein abgewiesen, um keine „undifferenzierte, massenhafte Wegsperrung der ,Irren "'27 zu praktizieren.

Im 19. Jahrhundert sah sich die Irrenheilanstalt einem Wandel unterzogen: Ausgehend von einem Ort der reinen Verwahrung transformierte sich die Anstalt zu einem Ort der Heilung, der für eine soziale Reintegration Sorge tragen sollte. ${ }^{28}$ Gerade in der ersten Hälfte des 19. Jahrhunderts setzte man auf das Modell der Anstalt als hierarchisch gegliedertes System, das auf den Ideen „Von Hausökonomie in der Vormoderne und schichtspezifische[n] Sozialordnungen"29 beruhte. Die Irrenanstalten wurden nach einem paternalistischen Modell geführt und organisiert. ${ }^{30}$ Am Ende des 19. Jahrhunderts folgte man dem „Programm" der staatlichen Fürsorge der "Geisteskranken“ in geschlossenen Anstalten, das aus einem „sicherheits-politischen Interesse“31 hervorging. Die Anstaltspsychiatrie wurde somit vermehrt auf die "gefährlichen Irren" mit dem Zweck einer zumeist lebenslängen Unterbringung ausgerichtet. ${ }^{32}$

\section{Die Psychiatrie als Totale Institution}

\section{Die Totale Institution - Eine Definition}

Dieses Kapitel beschäftigt sich mit dem von Erving Goffman geprägten Begriff der "Totalen Institution", den er ausführlich in seinem bereits vorgestellten Werk "Asylums" festgehalten hat. Gemäß Goffman ist die "Totale Institution" ein Wohn- oder Arbeitsplatz. An diesem führt eine große Anzahl von ähnlich gestellten Individuen, die über einen längeren Zeitraum von der breiten Gesellschaft abgeschnitten ist, ein geschlossenes und formal kontrolliertes Leben. ${ }^{33}$ Als charakteristisches Beispiel verweist der Soziologe auf das Gefängnis, in dem einem streng reglementierten Alltag nachgegangen wird. ${ }^{34}$ Genauso können aber auch andere totale Institutionen dazugezählt werden, deren Alltag von diesen Reglementierungen bestimmt sind, wie beispielsweise psychiatrische Kliniken, die den Schwerpunkt von Goffmans Feldforschung bildeten. Mithilfe der teilnehmenden Beobachtung entwickelte er ein „innerperspektivisches, mikroso-

26 Maria Heidegger/Oliver Seifert, „Nun ist aber der Zweck einer Irrenanstalt Heilung.... . Zur Positionierung des „Irrenhauses" innerhalb der psychiatrischen Landschaft Tirols im 19. und frühen 20. Jahrhundert, in: Elisabeth DietrichDaum/Rodolfo Taiani (Hrsg.), Psychiatrielandschaft, Innsbruck-Wien-Bolzen 2008, S. 24-46, hier S. 31.

27 Heidegger/Seifert, Zweck einer Irrenanstalt, S. 31.

28 Maria Heidegger, Psychiatrische Pflege in der historischen Anstalt. Das Beispiel der "k.k. Provinzialanstalt” Hall in Tirol 1830-1850, in: Erna Appelt/Maria Heidegger u.a. (Hrsg.), Who Cares? Betreuung und Pflege in Österreich. Eine geschlechterkritische Perspektive, Innsbruck 2010, S. 87.

29 Heidegger, Psychiatrische Pflege, S. 87-97, hier S. 87

30 Ralser, Subjekt, S. 158.

31 Astrid Ley, Psychiatriekritik durch Psychiater, Sozialreformerische und professionspolitische Ziele der Erlanger Anstaltsdirektors Gustav Kolb (1870-1938), in: Fangerau/Nolte (Hrsg.), Moderne Anstaltspsychiatrie, S. 199.

32 Ley, Psychiatriekritik, S. 199; Christian Müller, Heilanstalt oder Sicherungsanstalt? Die Unterbringung geisteskranker Rechtsbrecher als Herausforderung der Anstaltspsychiatrie im Deutschen Kaiserreich, in: Fangerau/Nolte (Hrsg.), Moderne Anstaltspsychiatrie, S. 103-115, hier S. 103.

33 Goffman, Asylums, S. 11.

34 Ebd. 
ziologisch orientiertes Modell von geschlossenen Institutionen, die sich mit der Verwahrung von Personen beschäftigten".35

Der totale Charakter, mit dem alle Institutionen gekennzeichnet sind, lässt sich durch einschränkende Faktoren, wie Abschottung zur Außenwelt, geschlossene Türen, hohe Mauern oder Stacheldraht, etc. versinnbildlichen. ${ }^{36}$ Institutionen, die diese freiheitsraubenden Einschränkungen aufweisen, betitelt Goffman als totale Institutionen, die in fünf Gruppen differenziert werden können: Fürsorgeanstalten wie Waisen- oder Armenfürsorgehäuser, Anstalten wie Tuberkulose-Sanatorien oder psychiatrische Kliniken, Institutionen, die zum Wohlergehen der Gesellschaft und des Staates potenziell bedrohliche Personen in Verwahrung nehmen (z.B. Gefängnisse), aber auch Institutionen, die für einen reibungslosen Arbeitsablauf sowie Zucht und Ordnung sorgen (wie Kasernen, Internate, Arbeitslager) sowie religiöse Refugien (unter anderem Abteien, Klöster). ${ }^{37}$

Des Weiteren sind totale Institutionen geprägt von einem wechselseitigen Verhältnis zwischen Insassinnen und Insassen sowie Personal. Auch in der psychiatrischen Anstalt hängt die Rolle einer jeden Gruppe (die Patientengruppe auf der einen und die des Personals auf der anderen Seite), die in dem System „Totale Institution" agiert, von der jeweiligen Verordnung ab. Die Verhaltensweisen sind vorgegeben und müssen strikt befolgt werden. Dabei stehen die einzelnen Individuen einer Gruppe (Patienten) in Relation zu jenen agierenden Subjekten (Anstaltspersonal) der anderen. Bei diesen Beziehungen handelt es sich meist um zeitlich begrenzte ",Zwangsbeziehungen', in denen das soziale Gefälle zwischen den Akteurlnnen zu asymmetrischen Abhängigkeits- und Machtbeziehungen [führt]"38

Zudem geht Goffman davon aus, dass in der modernen Gesellschaft das zentrale Merkmal "Totaler Institutionen“ in der Aufhebung der Barrieren, die die drei Lebensbereiche (Schlaf-, ,Spiel“- und Arbeitsplatz) normalerweise trennen, gefunden werden kann ${ }^{39}$ :

Erstens: Alle Aspekte des alltäglichen Lebens werden am gleichen Ort und unter derselben Autorität durchgeführt.

Zweitens: Die Arbeitsabläufe der Institutionsmitglieder werden in Gesellschaft ihrer Genossen ausgeführt, wobei alle gleich behandelt werden und dieselbe Arbeit verrichten.

Drittens: Alle täglichen Aufgabenbereiche, die aus vorherigen Tätigkeiten herausfolgen, sind streng geplant, und werden durch formale Reglementierungen genau vorgegeben und durch Obrigkeiten kontrolliert/überwacht.

35 Martin Scheutz, "Totale Institutionen“ - missgeleiteter Bruder oder notwendiger Begleiter der Moderne? Eine Einführung, in: Ders. (Hrsg.), Totale Institutionen (Wiener Zeitschrift zur Geschichte der Neuzeit 8/1), Innsbruck 2008,

S. 3-19, hier S. 4.

36 Goffman, Asylums, S. 15

37 Ebd., S. 16.

38 Dietrich-Daum/Heidegger, Menschen in Institutionen, S. 44.

39 Goffman, Asylums, S. 17. 
Viertens: Die unterschiedlich forcierten Arbeitsabläufe werden zu einem einzigen "rationalen Plan“ zusammengefügt, zur Zweckerfüllung der offiziellen Institutionsziele. ${ }^{40}$

Im nachfolgenden Abschnitt sollen diese vier Merkmale, die nach Goffman eine "Totale Institution" ausmachen, am Beispiel der Provinzial-Irren-Heilanstalt Hall in Tirol untersucht werden. Inwieweit lassen sich die Merkmale in Hall wiederfinden, sodass die Anstalt als "Totale Institution" bezeichnet werden kann?

Aspekte und Merkmale der Totalen Institution am Beispiel der Irren-Heil-Anstalt Hall in Tirol

Unter Zuhilfenahme der 1842 publizierten „Beschreibung“ über die Haller Anstalt vom damaligen Anstaltsdirektor und Primararzt ${ }^{41}$ Dr. Johann Tschallener sollen als nächstes die von Goffman herausgearbeiteten Aspekte und Merkmale einer "Totalen Institution“ am Beispiel der Haller Anstalt untersucht werden.

Ihre Pforten öffnete die „k.k. Provinzial-Irren-Heilanstalt" Hall in Tirol am 1. September $1830^{42}$ unter der Leitung von Dr. Anton Pascoli (1788-?). Ab 1834 trat Dr. Johann Tschallener (1783-1855) bis zum Jahre 1854 dessen Nachfolge an. ${ }^{43}$ Diese Zeitspanne umfasst auch den ungefähren Untersuchungszeitraum (1830-1900) des nachfolgenden Abschnitts.

Bereits mit dem Eintritt des Individuums in das Anstaltsgebäude zeigt sich der totale Charakter, den die Anstalt aufweist, und erste freiheitsraubende Einschränkungen treten in Kraft: Patientinnen und Patienten werden von der Außenwelt abgeschnitten. Diese Trennung zur Außenwelt geht mit einem Rollenverlust einher, indem der Patient/die Patientin ein gewisses Aufnahmeprocedere durchlaufen muss und ihm/ihr das persönliche Hab und Gut genommen wird. ${ }^{44}$ So heißt es in Tschalleners „Beschreibung”, dass das ankommende Individuum in Gegenwart des Wartpersonals und des Sekundär- sowie Hauswundarztes untersucht, gewaschen und nach Einkleidung (entweder mit eigener oder der Anstaltskleidung) und Bestimmung der Diätklasse in die Obhut des zuständigen Wartpersonals gegeben wird ${ }^{45}$, wobei angemerkt werden muss, dass es sich beim Wartpersonal bzw. dem „Irrenwärter" im 19. Jahrhundert um keinen Ausbildungsberuf handelte. ${ }^{46}$ Der Aufnahmeprozess kann gemäß Goffman eher als „, trimming' or, programming ${ }^{\prime \prime 47}$ angesehen werden, denn durch seine Isolierung verliert das Individuum ein Stück weit seine Identität, und es wird zu einem Objekt der Verwaltungsmaschinerie der Anstalt gemacht. ${ }^{48}$

40 Goffman, Asylums, S. 17.

41 Der Direktor war zugleich Primararzt. Ihm oblag der Oberaufsicht und Leitung der Anstalt: Tschallener, Beschreibung, S. 41, §17.

42 Heidegger/Seifert, Zweck einer Irrenanstalt, S. 26.

43 Institut der Geschichtswissenschaften und Europäische Ethnologie und Institut für Erziehungswissenschaften UIBK, Psychiatrische Landschaften, 2009, [http://pschiatrische-landschaften.net/Biografien_der_Direktoren_und_ anderer_Angeh\%C3\%B6riger_des_Anstaltspersonals], eingesehen 1.2.2015.

44 Goffman, Asylums, S. 25 f.

45 Tschallener, Beschreibung, S. 19.

46 Dietrich-Daum/Heidegger, Menschen in Institutionen, S. 56.

47 Goffman, Asylums, S. 26.

48 Ebd. 
Auch andere einschränkende Faktoren, die Goffman in seinem Aufsatz aufzählt, lassen sich laut der Beschreibung von Tschallener in Hall wiederfinden. So geht daraus hervor, dass dem „Irren“ die physische Freiheit in einem gewissen Maße gewährt wird, sodass er nicht ständig eingesperrt oder angekettet sei. Weiters ist die Rede einer "verlässlichen[n] Versicherung", ${ }^{49}$ die in eine äußere und innere differenziert wurde. Bei der äußeren handelte es sich zum Beispiel um Einfriedungsmauern, die eine Höhe von acht Schuh (ca. 2,52 m) aufweisen mussten. Des Weiteren wurde darauf geachtet, dass es in den Hofräumen keine Gegenstände gab, die ein Emporklettern ermöglicht hätten. ${ }^{50}$ Unter der inneren Versicherung verstand man die besondere Rücksichtnahme auf jene PatientInnen, die nicht zur gleichen Zeit mit den anderen „Irren“ ins Freie durften. Ihnen wurde der Ausgang durch eine eigene Tagwache, bestehend aus zwei Wärtern, die im Wechsel Aufsicht hatten, versperrt. ${ }^{51}$ Ebenso legte man großen Wert auf die Sicherheit in den „Irrenzimmern“. Die Türen waren mit einem speziellen Schloss und einer Queröffnung zur Beobachtung der „Irren“ versehen, und die vergitterten Fenster hatten sogenannte Fenstersperren mit Eisenstäben. Zusätzlich waren schließbare Balken daran angebracht, um ein Einschlagen der Fenster zu verhindern. ${ }^{52}$

Als nächstes werden die im vorherigen Kapitel beschriebenen vier Merkmale einer „Totalen Institution" bezugnehmend auf die Haller Anstalt skizziert: Goffmans Ausführungen zufolge umfasste der Arbeitsbereich des Personals in totalen Institutionen vielmehr die Funktion des Überwachens als die der Führung..$^{53}$ Eine Form der Überwachung stellte einerseits die Kommunikationskontrolle ${ }^{54}$ dar, die auch in der Haller Anstalt Anwendung gefunden hat, indem vom Stab bestimmt wurde, an und wie oft PatientInnen Besuch erhalten durften. Es war vorgegeben, dass die Besucherlnnen aus naher Umgebung sein mussten (ergo keinen von der Anstalt weit entfernten Wohnsitz besitzen durften) und erst nach Bekanntgabe der Direktion an der Eingangspforte der Heilanstalt empfangen und vom Hauswundarzt herumgeführt wurden. ${ }^{55}$ Die Besucherlnnen waren dazu verpflichtet sich in ein sogenanntes "Passantenbuch"56 (Name, Charakter, Geburtsort) einzutragen, woraufhin der Direktor die Erlaubnis zum Betreten der Anstalt erteilte. ${ }^{57}$ Andererseits spiegelte sich die permanente Überwachung in der Hausordnung der Anstalt wider. Sie hatte eine detaillierte Zusammenstellung von Regeln zum Inhalt, an die sich sowohl InsassInnen als auch Wartpersonal halten mussten. Diese Verordnung gab den exakten Tagesablauf wieder. ${ }^{58}$ In ihr lassen sich dabei die ersten drei Merkmale der totalen Institution (alle alltagsbezogenen Faktoren finden am gleichen Ort unter derselben Autorität statt, die Arbeitsabläufe der Institutionsmitglieder werden in Gesellschaft ihrer Genossen ausgeführt, und die Aufgabenbereiche sind

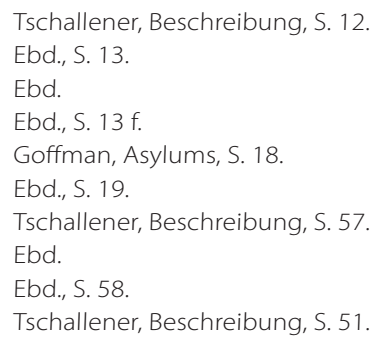


präzise vorgegeben und werden von einer Obrigkeit überwacht) wiederfinden. Diese werden im Folgenden anhand der Haller Tagesordnung zusammenfassend erläutert:

\begin{tabular}{|c|c|c|}
\hline Tag & Vormittägige Beschäftigungen & Nachmittägige Beschäftigungen \\
\hline So & $\begin{array}{l}\underline{06.00-07.30} \\
\text { Aufstehen, Morgengebet, Aufbetten und } \\
\text { Zimmerfegen } \\
\underline{07.30-08.00} \\
\text { Frühstück (Speisesaal) } \\
\underline{08.00-08.30} \\
\text { Heilige Messe } \\
\underline{08.30-10.00} \\
\text { Ärztliche Ordination } \\
\underline{10.00-11.00} \\
\text { Bewegung im Freien } \\
\frac{11.00-11.30}{\text { Mittagessen (Speisesaal) }}\end{array}$ & $\begin{array}{l}\frac{13.30-14.00}{\text { Rosenkranzandacht }} \\
\frac{14.30-16.00}{\text { Bewegung im Freien }} \\
\frac{16.00-17.30}{\text { Singschule }} \\
\frac{17.30-18.00}{\text { Abendessen (Speisesaal) }} \\
\frac{18.00-19.00}{\text { Spielveranstaltungen (Speisesaal) }}\end{array}$ \\
\hline Mo & $\begin{array}{l}\frac{06.00-10.00}{\text { Wie am Sonntag }} \\
\frac{10.00-11.00}{\text { Religionsunterricht für Deutsche }} \\
\text { Unterricht des Wartpersonals über die } \\
\text { Krankenpflege } \\
\frac{11.00-11.30}{\text { Mittagessen (Speisesaal) }}\end{array}$ & $\begin{array}{l}\frac{13.00-14.30}{\text { Schneider-, Schuster- und Tischlerarbeiten }} \\
\text { (Werkstätte) } \\
\text { Schreibschule (Speisesaal) } \\
\frac{14.30-15.30}{\text { Bewegung im Freien }} \\
\frac{15.30-16.00}{\text { Rosenkranzandacht }} \\
\frac{16.00-17.30}{\text { Singschule }} \\
\frac{17.30-18.00}{\text { Abendessen (Speisesaal) }} \\
\frac{18.00-19.00}{\text { Spielveranstaltungen (Speisesaal) }}\end{array}$ \\
\hline Di & $\frac{06.00-11.30}{\text { Wie am Sonntag }}$ & $\begin{array}{l}\frac{13.00-14.30}{\text { Wie am Montag }} \\
\text { Statt Schreibschule Leseübungen } \\
\frac{14.30-16.00}{\text { Bewegung im Freien }} \\
\frac{16.00-17.30}{\text { Singschule }} \\
\frac{17.30-19.00}{\text { Wie am Montag }}\end{array}$ \\
\hline Mi & $\frac{06.00-11.30}{\text { Wie am Sonntag }}$ & $\frac{13.00-14.30}{\text { Wie am Montag }}$ \\
\hline Do & $\frac{06.00-11.30}{\text { Wie am Sonntag }}$ & $\frac{13.00-19.00}{\text { Wie am Dienstag }}$ \\
\hline
\end{tabular}




\begin{tabular}{|c|c|c|}
\hline $\mathrm{Fr}$ & $\begin{array}{l}\frac{06.00-10.00}{\text { Wie am Sonntag }} \\
\frac{10.00-11.00}{\text { Religionsunterricht für Italiener }} \\
\text { Diverse Hausarbeiten für Deutsche } \\
\frac{11.00-11.30}{\text { Mittagessen (Speisesaal) }}\end{array}$ & $\begin{array}{l}\frac{13.00-14.30}{\text { Schneider-, Schuster- und Tischlerarbeiten }} \\
\text { (Werkstätte) } \\
\text { Schreibschule (Speisesaal) } \\
\frac{14.30-15.30}{\text { Zimmerreinigung }} \\
\frac{15.30-16.00}{\text { Rosenkranzandacht }} \\
\frac{16.00-17.30}{\text { Zimmerreinigung }} \\
\frac{17.30-18.00}{\text { Abendessen (Speisesaal) }}\end{array}$ \\
\hline $\mathrm{Sa}$ & $\begin{array}{l}\frac{06.00-10.00}{\text { Wie am Sonntag }} \\
\frac{10.00-11.00}{\text { Zimmerreinigung }} \\
\frac{11.00-11.30}{\text { Mittagessen (Zimmer) }}\end{array}$ & $\begin{array}{l}\frac{13.00-14.30}{\text { Schneider-, Schuster- \& Tischlerarbeiten }} \\
\text { (Werkstätte) Schreibschule (Speisesaal) } \\
\frac{14.30-17.00}{\text { Zimmerreinigung }} \\
\frac{17.30-18.00}{\text { Abendessen (Zimmer) }}\end{array}$ \\
\hline
\end{tabular}

Abbildung 1: Tagesordnung für die Männer der Provinzial-Irrenheilanstalt Hall/Tirol (Wintermonate)59

Bei der Tagesordnung unterschied man zwischen männlichen und weiblichen Insassen, da die Anstalt, wie andere Einrichtungen zu dieser Zeit auch, eine geschlechterspezifische Trennung vornahm. ${ }^{60}$ Die in dieser Arbeit abgebildete Tagesordnung stellt den Ablauf einer ganzen Woche für die männlichen Insassen der Haller Anstalt während der Wintermonate dar. Wie aus der Tabelle hervorgeht, begann der Tag um 06.00 Uhr morgens, gefolgt vom Morgengebet, dem Aufbetten sowie dem Zimmerfegen. Nachdem um halb acht das Frühstück im Speisesaal eingenommen wurde, besuchten die männlichen Insassen samt dem Wartpersonal die Heilige Messe in der anstaltseigenen Kapelle. Daraufhin erfolgte die allvormittägliche Ordination, die bei den Männern im Gegensatz zu den Frauen, die den Arzt auf ihren Zimmern empfingen, ${ }^{61}$ im ärztlichen Ordinationsraum stattfand. Der morgendliche Ablauf bis 10.00 Uhr blieb an allen Tagen gleich. Nach 10.00 Uhr gab es unterschiedliche Aktivitäten, wie beispielsweise die Zimmerreinigung am Samstag oder den getrennten Religionsunterricht von deutsch- und italienischsprachigen Patienten am Montag sowie am Freitag. Am Nachmittag ging das Beschäftigungsprogramm weiter, indem unter anderem die Teilnahme zur Andacht, Spaziergänge im Garten/Hof und an manchen Tagen Schreib- und Leseübungen vorgesehen waren. Gerade das Lesen war in den Anstalten eine beliebte und oft angewandte Methode, die nicht nur als „Beschäftigung, [zur] Bildung [und]

59 Bei der in Tschalleners Beschreibung abgebildeten Originaltabelle befindet sich kein Datum. Da die Beschreibung jedoch 1842 publiziert wurde, kann davon ausgegangen werden, dass die Tagesordnung frühestens zu Tschalleners Amtsantritt (1834) und spätestens bis 1842 gültig gewesen ist. Tschallener, Beschreibung, S. 46 f.

60 Dietrich-Daum/Heidegger, Menschen in Institutionen, S. 58.

61 Tagesordnung für die Frauen (Wintermonate), in: Tschallener (Hrsg.), Beschreibung, S. 48 f. 
[dem] Vertreiben von ,morbiden Gedanken"'62 dienen sollte, sondern den InsassInnen und dem Wartpersonal auch die Chance bot sich über die Belletristik auszutauschen. Tschallener selbst verweist in seiner Beschreibung auf die hauseigene Bibliothek der Anstalt, die, was die Bücheranzahl betrifft, jedoch "zu wünschen übrig lässt"63 Des Weiteren mussten sowohl die weiblichen als auch die männlichen Insassen diverse Arbeiten verrichten. Bei den Frauen handelte es sich um für diese Zeit typische Hausfrauenarbeiten, denen sie im Arbeitszimmer nachgingen. ${ }^{64}$ Diese Arbeiten reichten vom „Stricken, Spinnen, Nähen [...], [über] [das] Putzen [...] [bis hin] [zur] Mithilfe in der Küche bzw. bei der Wäsche“"65 unter Aufsicht des weiblichen Wartpersonals. Die Männer hingegen mussten in der Werkstatt verschiedene Schneider-, Schuster- und Tischlerarbeiten ausführen, wie aus der Tabelle zu entnehmen ist. Man ließ die PatientInnen nicht ohne Grund arbeiten, denn Arbeit wurde seit 1800 nicht nur als „Teil der Therapie"66 angesehen, sondern zugleich mit dem aus der absolutistischen Epoche wurzelnden "Nützlichkeitsgedanken"67 in Verbindung gesetzt. Auch wenn die Patienten und Patientinnen "für ihre Arbeit nach Maßgabe ihrer Verwendung [...] belohnt w[u]rden"68 kann die Vermutung aufgestellt werden, dass der Arbeitseinsatz der InsassInnen nicht nur als Beschäftigungs- und Heilungsmethode diente, sondern auch zur Personalkosteneinsparung und Finanzierung der Anstalt, da die PatientInnen eine weit geringere Entlohnung erhielten als das Personal. ${ }^{69}$

Abschließend kann gesagt werden, dass anhand der Tagesordnung ersichtlich wird, dass es durch die minutiös vorgegebenen Abläufe zur Verletzung der Autonomie des Handelnden selbst kam, dass jeder Schritt der InsassInnen überwacht und bei Verstoß gegen die Regeln durch Sanktionen der Funktionsträger bestraft wurde. ${ }^{70}$ Man sprach von disziplinierenden Maßnahmen, die zum Einsatz kamen, um andere Institutionsmitglieder zu schützen und „einen, normalen' Anstaltsbetrieb [...] gewährleisten [zu können]"71 In der "Beschreibung" der Haller Irren-Heilanstalt steht beispielhaft, dass „von den Strafen in einer Irrenanstalt niemals ganz Umgang genommen werden kann",72 jedoch oblag dem Direktor als einzigem die Veranlassung von Strafen, "hiebei [sollte] aber mit aller Klugheit und mit Vermeidung eines jeden Scheines von Leidenschaft vorzugehen [sein], damit diese moralischen Heilmittel ihren Zweck nicht verfehlen, und die Uebel [sic!] nicht ärger machen".73

62 Ursula A. Schneider/Annette Steinsiek, „,Die Lektüre der Pfleglinge“. Ein literaturwissenschaftlicher Blick auf die historische Bibliothek des Psychiatrischen Krankenhauses Hall, in: Dietrich-Daum et al. (Hrsg.), Psychiatrische Landschaften, S. 100.

63 Tschallener, Beschreibung, S. 7.

64 Tagesordnung für die Frauen (Wintermonate), in: ebd., S. 48 f.

65 Maria Heidegger, Maria M. Das Dienstmädchen, in: Heidegger et al, Ich lasse mich, S. 226.

66 Ebd., S. 210.

67 Ebd.

68 Tschallener, Beschreibung, S. 5.

69 Ebd., S. 6.

70 Goffman, Asylums, S. 43.

71 Marietta Meier, Zwang und Autonomie in der psychiatrischen Anstalt. Theoretische Überlegungen und empirische Befunde aus historischer Sicht, in: Wulf Rössler/Paul Hoff (Hrsg.), Psychiatrie zwischen Autonomie und Zwang, Heidelberg 2005, S. 69-87, hier S. 76.

72 Tschallener, Beschreibung, S. 57.

73 Ebd. 
Bevor dieser Teil der Arbeit mit dem letzten Merkmal "Totaler Institutionen" abschließt, soll noch kurz auf die Frage eingegangen werden, wie die Patientlnnen den durch Reglementierung streng vorgegebenen Anstaltsalltag empfunden haben. Hierzu lassen sich viele „Egodokumente" finden, die Aufschluss auf die Gefühlslage der InsassInnen geben. ${ }^{74}$ Um den Rahmen dieser Arbeit jedoch nicht zu sprengen, müssen hierfür drei ausgewählte Beispiele aus dem Begleitbuch zur Ausstellung „Ich lasse mich nicht länger für einen Narren halten" genügen.

So berichtet die Historikerin Maria Heidegger von dem Fall des Patienten Franz S. aus dem Jahre 1834. Dieser litt als Dritte-Klasse-Patient ${ }^{75}$ in Hall unter ständigem Hunger, da er nie mehr als eine „Drittel-Portion, bestehend aus Semmelbrot und zum Abendessen einer Suppe"76 erhielt. Erschwerenderweise gab es für Franz S. von Zeit zu Zeit überhaupt keine Nahrung, um ihn zu maßregeln. Zuerst reagierte er mit Zerstörungswut, später mit einem Hungerstreik, den er weniger schlimm empfand als seinen Freiheitsentzug, respektive das Wegsperren aus Strafe. ${ }^{77}$ Einen anderen Fall, den Heidegger untersuchte, stellte die 46-jährige Anna K. dar, die 1855 in die Haller Anstalt eingewiesen wurde, und durch mehrmalige Nahrungsverweigerung Widerstand gegen die Obrigkeit leistete. ${ }^{78}$ Ein Grund dafür fand sich unter anderem in ihrem Drang nach Freiheit beziehungsweise Entlassung, die sie nicht nur für sich, sondern ebenso für ihre "Leidensgenossen" einzufordern versuchte. ${ }^{79}$ Das dritte Beispiel handelt von Josef B., der aufgrund von chronischem Alkoholismus 1903 zuerst in die Psychiatrische Klinik Innsbruck und nur einen Monat später wie Franz S. als Dritte-Klasse-Patient nach Hall kam. Er schimpfte über Hunger sowie Kälte, über das Gefühl zu Unrecht eingesperrt worden zu sein und verkündete, dass er sich nicht länger für einen Narren halten lassen würde..$^{80}$

Anhand dieser drei Auszüge lässt sich bereits erkennen, dass die Patientlnnen weit davon entfernt waren, sich in der Anstalt "zu Hause" und somit wohl zu fühlen. Der instinktive Drang nach Freiheit, der den InsassInnen verwehrt wurde, ist allen drei gemeinsam. Dieses Freiheitsgefühl und die in ihrem Verständnis ungerechten disziplinierenden Maßnahmen mündeten in wiederholten Widerstandsaktionen durch die Patienten und Patientinnen. Zusätzlich beeinträchtigte das Hungerleiden den Gemütszustand von Franz S. und Josef B.

Zum Schluss soll nun das vierte Merkmal "Totaler Institutionen“ beleuchtet werden, nämlich, dass die unterschiedlich forcierten Arbeitsabläufe zu einem einzigen rationalen Plan zusammengefügt werden, um die offiziellen Institutionsziele bzw. primären Ziele erfüllen zu können: Das offizielle Ziel der Irren-Heilanstalt Hall bestand einerseits

74 Diverse Beispiele finden sich in: Heidegger et al. (Hrsg.), Ich lasse mich.

75 Man differenzierte zwischen Erste-, Zweite- und Dritte-Klasse-Patienten, wobei letztere in Hall gratis versorgt wurden. Tschallener, Beschreibung, S. $10 \mathrm{f}$

76 Maria Heidegger, Franz S. Klagte stets über Hunger, in: Heidegger et al. (Hrsg.), Ich lasse mich, S. 256.

77 Heidegger, Franz S., S. 258

78 Maria Heidegger, Anna K. 12.037 mal künstlich ernährt, in: Heidegger et al. (Hrsg.), Ich lasse mich, S. 266.

79 Ebd., S. 268.

80 Andreas Oberhofer, Josef B. Ich lasse mich nicht mehr für einen Narren halten, in: Heidegger et al. (Hrsg.), Ich lasse mich, S. 144-146. 
in der Pflege und der Heilung von Patienten, ${ }^{81}$ um sie anschließend wieder in die Gesellschaft eingliedern zu können, andererseits in der Verwahrung von "gefährlichen Irren"82 Auch wenn die Prämisse der Heilung geltend gemacht wurde, ging es aber in erster Linie um die "Abgrenzung ${ }^{\prime 83}$ zu anderen existierenden psychiatrischen Einrichtungen, die für eine Seelenheilung der Erkrankten weniger zielführend gewesen wären als die Irrenanstalt selbst. ${ }^{84}$ Als sekundäres, respektive verdecktes Institutionsziel kann die Sicherung der Gesellschaft angeführt werden, die insbesondere vor den "gefährlichen Irren" beschützt werden sollte. ${ }^{85}$ Die Historikerinnen Dietrich-Daum und Heidegger schreiben der Haller Anstalt eine "Zwischenposition zwischen ,Totaler Institution' und paternalistischer Fürsorgeeinrichtung "86 zu. Diese Aussage findet mit Abschluss dieser Untersuchung auf jeden Fall Zustimmung. Denn wie die herausgearbeiteten Ergebnisse in dieser Proseminararbeit verdeutlichen, trafen sowohl die freiheitseinschränkenden Aspekte als auch die vier Merkmale, die Goffman einer „Totalen Institution" zuschreibt, auf die Provinzial-Irren-Heilanstalt Hall zu. Was den paternalistischen Charakter der Fürsorgeeinrichtung angeht, weisen die beiden Historikerinnen auf die "zahlreichen Formulierungen über die Rolle des Personals oder der, Insassen "'"87 hin, die sich an „sprachliche Muster hausväterlicher Familienkonzeptionen"88 anlehnen.

\section{Fazit}

Die historische Entwicklung der Psychiatrie hat gezeigt, dass die üblichen Verwahranstalten des 18. Jahrhunderts allmählich durch Einrichtungen, die auf die Verwahrung und Heilung von „psychisch Kranken“ spezialisiert waren, abgelöst wurden. Den ausschlaggebenden Punkt bildete dabei die Idee, dass die Anstalt eine heilende Funktion übernehmen sollte. Hinzu kamen die disziplinierende und erzieherische Rolle, die die Verwahranstalt über ihre PatientInnen ausübte, um Heilung herbeizuführen.

Das, was solche "Orte der Verwahrung" ausmachte, versuchte Erving Goffman mithilfe seines Konzeptes der "Totalen Institution" zu beschreiben. Wie sich während den Untersuchungen in dieser Arbeit herausstellte, lassen sich die von Goffman genannten Aspekte anhand des Beispiels der Provinzial-Irren-Heilanstalt Hall in Tirol belegen. So weist die Haller Anstalt beispielsweise einen totalen Charakter durch einschränkende Faktoren auf, die wiederum durch die Abschottung der InsassInnen von der Außenwelt, die hohen Mauern sowie die gesicherten Türen und Fenster in den „Irrenzimmern“

81 Angela Griessenböck, Zur Geschichte, S. 122.

82 Dietrich-Daum/Heidegger, Die k.k. Provinzial, in: Scheutz (Hrsg.), Totale Institutionen, S. 68-85, hier S. 72.

83 Heidegger/Seifert, Zweck einer Irrenanstalt, S. 30.

84 Ebd.

85 RainerFiedl,Von den Irrenanstaltenzurmodernen Psychiatrie, in:Scheutz(Hrsg.),TotaleInstitutionen, S. 130-134, hier S. 133.

86 Dietrich-Daum/Heidegger, Hall im Vormärz, S. 84 f.

87 Ebd., S. 85

88 Ebd 
versinnbildlicht wurden. Durch diese Absonderung von der Außenwelt ging zusätzlich ein sozialer Rollenverlust einher und das Individuum verlor durch seine Isolierung nicht nur ein Stück weit seine Identität, sondern wurde zugleich zu einem Objekt der Verwaltungsmaschinerie der Anstalt gemacht.

Zudem treffen auch die vier Merkmale „Totaler Institutionen“ auf die Anstalt zu. Anhand der vorgestellten Tagesordnung für die Männer in Hall wurde ersichtlich, dass die ersten drei Merkmale in der Anstalt anzufinden sind, nämlich: Alle den Alltag betreffenden Aspekte finden am gleichen Ort und unter derselben Autorität statt. Die Arbeitsabläufe der Institutionsmitglieder werden in Gesellschaft ihrer GenossInnen ausgeführt und die Aufgabenbereiche sind streng geplant, genau vorgegeben und werden von einer Obrigkeit überwacht sowie kontrolliert. Sobald vom Tagesablauf abgewichen wurde, drohten den PatientInnen verschiedene Straf- und Disziplinierungsmaßnahmen. Außerdem sah die Tagesordnung verschiedene Arbeitsschritte vor, die die Patienten und Patientinnen für die Anstalt gegen einen geringen Lohn auszuführen hatten. Auch wenn die Arbeit als Teil des Heilkonzeptes gesehen wurde, setzte man die InsassInnen vermutlich als „billige Arbeitskräfte“ ein, um Personalkosten einsparen zu können.

Für die in der Anstalt untergebrachten Individuen bedeutete dies vor allem, ein Leben unter ständiger Fremdkontrolle führen zu müssen. Die drei Patientenbeispiele, die in dieser Arbeit genannt wurden, verdeutlichten dies.

Hinsichtlich des letzten Merkmales, das die Institutionsziele betrifft, verstand sich die Anstalt offiziell als ein Ort der Verwahrung und Heilung. Durch therapeutische und disziplinierende Methoden wie Zucht und Ordnung, aber auch Arbeit und Strafmaßnahmen sollte das Primärziel der Heilung und somit eine erfolgreiche Reintegration der geheilten PatientInnen in die Gesellschaft gewährleistet werden. Als sekundäres Ziel wurde die Sicherung der Gesellschaft gesehen. 


\section{Literatur}

Bretschneider, Falk/Scheutz, Martin/Weiß, Alfred Stefan, Machtvolle Bindungen - Bindungen voller Macht. Personal und Insassen in neuzeitlichen Orten der Verwahrung zwischen Konfrontation und Verflechtung, in: Falk Bretschneider/Martin Scheutz/Alfred Stefan Weiß (Hrsg.), Personal und Insassen von "Totalen Institutionen" - zwischen Konfrontation und Verflechtung (Geschlossene Häuser. Historische Studien zu Institutionen und Orten der Separierung, Verwahrung und Bestrafung 3), Leipzig 2011 , S. 7-24.

Chmielewski, Alexandra, Staat und Irrenfürsorge. Badische Psychiatrie-Reformen im 19. Jahrhundert, in: Zeitschrift für die Geschichte des Oberrheins 146 (1998), S. 437-453.

Dietrich-Daum, Elisabeth/Heidegger, Maria, Die k.k. Provinzial-Irren-Heilanstalt Hall in Tirol im Vormärz - eine Totale Institution?, in: Martin Scheutz (Hrsg.), Totale Institutionen (Wiener Zeitschrift zur Geschichte der Neuzeit 8/1), Innsbruck 2008, S. 68-85.

Dies./Heidegger, Maria, Menschen in Institutionen der Psychiatrie, in: Dietrich-Daum et al. (Hrsg.), Psychiatrische Landschaften, S. 43-68.

Dies./Kuprian, Hermann/Clementi, Siglinde/Heidegger, Maria/Ralser, Michaela, Psychiatrische Landschaften. Die Psychiatrie und ihre Patientinnen und Patienten im historischen Raum Tirol seit 1830, Innsbruck 2011.

Fiedl, Rainer, Von den Irrenanstalten zur modernen Psychiatrie, in: Martin Scheutz (Hrsg.), Totale Institutionen (Wiener Zeitschrift zur Geschichte der Neuzeit 8/1), Innsbruck 2008, S. 130-134.

Goffman, Erving, Asylums. Essays on the Social Situation of Mental Patients and other Inmates, London 1991.

Griessenböck, Angela, Zur Geschichte der psychiatrischen Landschaft im Kronland Tirol: Die "Landes-Irrenanstalten" in Hall in Tirol und in Pergine, in: Eberhard Gabriel/ Martina Gamper (Hrsg.), Psychiatrische Institutionen in Österreich um 1900, Wien 2009, S. 121-134.

Heidegger, Maria, Anna K. 12.037 mal künstlich ernährt, in: Heidegger et al. (Hrsg.), Ich lasse mich nicht, S. 266-271.

Dies./di Pauli, Cealia/Noggler, Lisa/Clementi, Siglinde/Ralser, Michaela/Dietrich-Daum, Elisabeth/Kuprian, Hermann (Hrsg.), Ich lasse mich nicht länger für einen Narren halten. Eine Ausstellung zur Geschichte der Psychiatrie in Tirol, Südtirol und im Trentino, Bozen 2012.

Dies., Franz S. Klagte stets über Hunger, in: Heidegger et al. (Hrsg.), Ich lasse mich, S. 256-265.

Dies./Noggler, Lisa, Historischer Blickwinkel. Blickwechsel in der Ausstellung, in: Heide- 
gger et al. (Hrsg.), Ich lasse mich, S. 10-13.

Dies., Maria M. Das Dienstmädchen, in: Heidegger et al. (Hrsg.), Ich lasse mich, S. 224231.

Dies./Seifert, Oliver, „Nun ist aber der Zweck einer Irrenanstalt Heilung...." Zur Positionierung des "Irrenhauses" innerhalb der psychiatrischen Landschaft Tirols im 19. und frühen 20. Jahrhundert, in: Geschichte und Region/Storia e Regione 17 (2008), Heft 2, S. 24-46.

Dies., Psychiatrische Pflege in der historischen Anstalt. Das Beispiel der „k.k. Provinzialirrenanstalt" Hall in Tirol 1830-1850, in: Erna Appelt/Maria Heidegger/Max Preglau/Maria A. Wolf (Hrsg.), Who cares? Betreuung und Pflege in Österreich. Eine geschlechterkritische Perspektive, Innsbruck 2010, S. 87-97.

Institut für Geschichtswissenschaften und Europäische Ethnologie und Institut für Erziehungswissenschaften UIBK, Psychiatrische Landschaften, 2009, [http://psychiatrischelandschaften.net/Biografien_der_Direktoren_und_anderer_Angeh\%C3\%B6riger_ des_Anstaltspersonals], eingesehen 1.2.2015.

Ley, Astrid, Psychiatriekritik durch Psychiater, Sozialreformerische und professionspolitische Ziele des Erlanger Anstaltsdirektors Gustav Kolb (1870-1938), in: Heiner Fangerau/Karen Nolte (Hrsg.), „Moderne Anstaltspsychiatrie im 19. und 20. Jahrhundert - Legitimation und Kritik (Medizin, Gesellschaft und Geschichte 26), Stuttgart 2006, S. 195-219.

Meier, Marietta, Zwang und Autonomie in der psychiatrischen Anstalt. Theoretische Überlegungen und empirische Befunde aus historischer Sicht, in: Wulf Rössler/Paul Hoff (Hrsg.), Psychiatrie zwischen Autonomie und Zwang, Heidelberg 2005, S. 69-87.

Müller, Christian, Heilanstalt oder Sicherungsanstalt? Die Unterbringung geisteskranker Rechtsbrecher als Herausforderung der Anstaltspsychiatrie im Deutschen Kaiserreich, in: Fangerau/Nolte (Hrsg.), Moderne Anstaltspsychiatrie, S. 103-115.

Oberhofer, Andreas, Josef B. Ich lasse mich nicht mehr für einen Narren halten, in: Heidegger et al, Ich lasse mich, S. 144-149.

Ralser, Michaela, Im Vordergrund die Klinik. Das Beispiel der Innsbrucker PsychiatrischNeurologischen Universitätsklinik um 1900, in: Geschichte und Region/Storia e Regione 17 (2008), Heft 2, S. 135-145.

Dies., Das Subjekt der Normalität. Das Wissensarchiv der Psychiatrie: Kulturen der Krankheit um 1900, München 2010.

Scheutz, Martin, "Totale Institutionen“ - missgeleiteter Bruder oder notwendiger Begleiter der Moderne? Eine Einführung, in: Martin Scheutz (Hrsg.), Totale Institutionen (Wiener Zeitschrift zur Geschichte der Neuzeit 8/1), Innsbruck 2008, S. 3-19. 
Schmiedebach, Heinz-Peter, "Zerquälte Ergebnisse einer Dichterseele”. Literarische Kritik, Psychiatrie und Öffentlichkeit um 1900, in: Heiner Fangerau/Karen Nolte (Hrsg.), "Moderne Anstaltspsychiatrie im 19. und 20. Jahrhundert - Legitimation und Kritik (Medizin, Gesellschaft und Geschichte 26), Stuttgart 2006,S. 259-281.

Schneider, Ursula A./Steinsiek, Annette, „Die Lektüre der Pfleglinge“. Ein literaturwissenschaftlicher Blick auf die historische Bibliothek des Psychiatrischen Krankenhauses Hall, in: Dietrich-Daum et al. (Hrsg.), Psychiatrische Landschaften, S. 99-107.

Shorter, Edward, Geschichte der Psychiatrie, Berlin 1999.

\section{Quelle}

Tschallener, Johann (Hrsg.), Beschreibung der k.k. Provincial-Irren-Heilanstalt zu Hall in Tirol mit Rücksicht auf die Statuten der Anstalt, auf die therapeutischen und psychologischen Grundsätze der Behandlung der Geisteskranken und auf ihre achtjährigen Resultate; mit 19 Krankengeschichten und verschiedenen Andeutungen zum Wohl dieser Unglücklichen; nebst einem Anhange über die Anlage von Zimmern für Irre und Tobende, Innsbruck 1842.

Dominique Karner ist Studentin der Geschichte (MA) im 3. Semester sowie studentische Mitarbeiterin am Institut für Geschichtswissenschaften und Europäische Ethnologie der Universität Innsbruck. dominique.karner@student.uibk.ac.at

\section{Zitation dieses Beitrages}

Dominique Karner, Totale Institutionen - Psychiatrien im 19. Jahrhundert am Beispiel der k.k. Provinzial-Irren-Heilanstalt Hall in Tirol, in: historia.scribere 8 (2016), S. 27-44, [http://historia.scribere.at], 2015-2016, eingesehen 14.6.2016 (=aktuelles Datum).

Creative Commons Licences 3.0 Österreich unter Wahrung der Urheberrechte der Autorlnnen. 
